Check for updates

Cite this: RSC Adv., 2017, 7, 41745

Received 28th June 2017

Accepted 21st August 2017

DOI: 10.1039/c7ra07172h

rsc.li/rsc-advances

\section{Synthesis and characterization of polylethylene terephthalate-co-1,4-cyclohexanedimethylene terephtlatate)-block-poly(tetramethylene oxide) copolymers $\uparrow$}

\author{
Sandra Paszkiewicz, (D) *a Anna Szymczyk, ${ }^{\text {b }}$ Daria Pawlikowska, ${ }^{a}$ Izabela Irska, ${ }^{a}$ \\ Iman Taraghi, ${ }^{\text {ac }}$ Ryszard Pilawka, de Jiali Gu, ${ }^{f}$ Xiaohong Li, ${ }^{f}$ Yingfeng Tu (D) ${ }^{f}$ \\ and Elzbieta Piesowicz ${ }^{a}$
}

A series of poly(ethylene terephthalate-co-1,4-cyclohexanedimethanol terephthalate)-blockpoly(tetramethylene oxide) (PETG-block-PTMO) copolymers were synthesized by means of a polycondensation process and characterized using ${ }^{1} \mathrm{H}$ nuclear magnetic resonance (H NMR) and Fourier transform infrared spectroscopy (FTIR), that confirm the successful synthesis of the material. Differential scanning calorimetry (DSC), small - and wide-angle X-ray diffraction (SAXS and WAXS), and thermogravimetric analysis (TGA) were used in order to evaluate the influence of the block copolymers' composition and microstructure on the phase transition temperatures, thermal properties, as well as the thermooxidative and thermal stability of the PETG-block-PTMO copolymers, respectively. The mechanical properties were investigated by tensile testing and dynamic mechanical measurements (DMTA). We found that along with an increase in PTMO weight fraction, both number-average molecular weights and intrinsic visocisities increase. Moreover, an increase in the flexible segments content in PETG-block-PTMO resulted in shifting the values of glass transition temperatures toward lower ones, which was confirmed by DSC and DMTA analyses, thus affirming the miscibility of both phases. At the same time, along with an increase of PTMO flexible segments amount in the PETG-block-PTMO copolymers, the values of Young's modulus, tensile strength at yield and weight losses in lower temperatures range, i.e. $280-390{ }^{\circ} \mathrm{C}$, decrease.

\section{Introduction}

Multi-blocks segmented poly(ether-ester)s (PEE)s have been extensively used for industrial applications where high strength and flexibility over a wide temperature range are desired. ${ }^{1}$ The increasing attention of R\&D groups is due to a combination of rubber-like properties in the solid-state and melt processability. ${ }^{2}$ Many of these materials are composed of a rigid "hard" segment with a high glass transition temperature $\left(T_{\mathrm{g}}\right)$ and

${ }^{a}$ West Pomeranian University of Technology, Institute of Materials Science and Engineering, Piastow 19 Av., 70310 Szczecin, Poland. E-mail: sandra.paszkiewicz@ zut.edu.pl

${ }^{b}$ West Pomeranian University of Technology, Institute of Physics, Piastow 48 Av., 70311 Szczecin, Poland

'Semnam University, Deparment of Mechanical Engineering, Semnam 35131-19111, Iran ${ }^{d}$ West Pomeranian University of Technology, Polymer Institute, Pulaskiego str. 10, 70322 Szczecin, Poland

${ }^{e}$ New Era Materials Sp. z o.o., Komandosow str. 1/7, 32085 Modlniczka, Poland ${ }^{f}$ College of Soochow University, Suzhou 215123, China

$\dagger$ Electronic supplementary information (ESI) available. See DOI: $10.1039 / \mathrm{c} 7 \mathrm{ra} 07172 \mathrm{~h}$ a rubbery "soft" segment with low $T_{\mathrm{g} .}{ }^{2}$ The best example of this type of material is Hytrel $®$ (Dupont), a family of compounds where the rigid segment is based on poly(butylene terephthalate) (PBT) and the rubbery segment is poly(tetramethylene oxide) (PTMO) (PBT-block-PTMO). The apparent cross-linked behaviour of the solid-state matrix is the result of crystalline domains of PBT, which crystallize from the melt to form physical crosslinks for the soft segments as well as any crystallized hard segment material. ${ }^{3}$ Moreover, the material, which might exhibit similar properties and thus could have the same applications as Hytrel ${ }^{\circledR}$ is block copolymer based on poly(ethylene terephthalate) (PET) as rigid segment (PET-blockPTMO). ${ }^{4}$ Additionally, since DuPont Tate \& Lyle started to commercially manufacture 1,3-propanediol (bio-PDO, Susterra $^{\mathrm{TM}}$, Zemea $\left.{ }^{\mathrm{TM}}\right){ }^{5}$ a new member of PEEs' group, based on poly(trimethylene terephthalate) (PTT) as the rigid segment, has been synthesized and characterized by Szymczyk et al. ${ }^{6}$ The overall features of the aforementioned PEEs depend on the chemical compatibility of hard and soft blocks and showed at least two-phase separated microstructures. For instance, in PET-block-PTMO, the phase separation occurs by the 
crystallization process of PET segments within amorphous PTMO chains. ${ }^{7}$ In turn, the study on the phase structure of PTTblock-PTMO showed two relaxation peaks corresponding to two glass transition temperatures of amorphous flexible polyether phase (PTMO) and rigid polyester phase (PTT). ${ }^{6}$ Therefore, it is of great importance to make the interfacial tension between rigid and soft phases stronger and thus to enhance the elastic properties of PEEs. If the hard and soft segments are miscible, one $T_{\mathrm{g}}$ could be observed. ${ }^{8}$ In this case, $T_{\mathrm{g}}$ increases with the increase of hard segment content. ${ }^{8}$

The cyclohexanedimethanol ([4-(hydroxymethyl) cyclohexyl] methanol, IUPAC) (CHDM) is a colourless solid glycol for modification of fibres and polyesters. Recently, Gue et al., investigated a new approach for preparation of CHDM via hydrogenation of bis(2-hydroxyethylene terephthalate) (BHET) obtained from waste PET. The incorporation of various content of CHDM within aromatic polyesters can lead to the development of different kinds of glycol modified co-polyesters with properties ranging from amorphous to highly crystalline. ${ }^{3}$ Poly(ethylene terephthalate)-co-1,4-cylclohexylenedimethylene terephthalate (PECT) is a non-crystalline amorphous copolyester that can be synthesized using terephtalic acid (TPA) (or dimethyl terephthalate) (DMT), ethylene glycol (EG) and CHDM (up to 50 mol\%). ${ }^{3,11}$ This glycol modified PET (PETG) exhibits unique advantages of clarity, high strength, low shrinkage, high dimensional stability and solvent resistance. ${ }^{\mathbf{1 2 - 1 5}}$ One can apply it for transparent decoration parts, food and water packaging, medical, automobile, thin films and polymer foils. ${ }^{16-19}$

Polymer foils have been widely used in solar photovoltaic systems, constructions and buildings (cushions), embossing structures, food packaging and medical instruments. ${ }^{20,21}$ Polymer foil's thickness is ranging from $0.5 \mu \mathrm{m}$ up to $1 \mathrm{~mm}$. PET, ${ }^{22}$ polyfluoroethylene propylene (FEP), ${ }^{23,24}$ polytertrafluoroethylene (TFE), ${ }^{25}$ ethylene tetra fluoro ethylene (ETFE), ${ }^{26,27}$ polycarbonate, ${ }^{28}$ nylon $6,6,{ }^{29}$ PETG,${ }^{30}$ etc. are some examples of the most commonly used polymer foils. Among them, PETG films have been performed in alkaline battery packing due to their excellent chemical resistance. ${ }^{31}$ Furthermore, Lee et al. $^{32}$ employed PETG sheet for the concentrators due to its low glass transition temperature $\left(81^{\circ} \mathrm{C}\right)$, that made it possible to shape by simultaneously applying heat and vacuum. Similarly to PET, the PETG also exhibits very low notched impact strength and can be treated as a rigid brittle polymer. ${ }^{33}$ This poor elasticity of PETG can be improved by introducing a flexible PTMO segment into the chain. Therefore, the goal of this study is to synthesize and characterize the new type of PEEs containing rigid segments of PETG and soft segments of PTMO with excellent elastic properties. The composition and chemical structure of the series of block copolymers were determined by ${ }^{1} \mathrm{H}$ quantitative nuclear magnetic resonance (NMR) and Fourier transform infrared spectroscopy (FTIR). The temperatures corresponding to phase transition changes were characterized by differential scanning calorimetry (DSC) and dynamic mechanical thermal analysis (DMTA). The crystallization behaviour of thin PETG-blockPTMO films were characterized by small-angle and wide-angle $\mathrm{X}$-ray scattering (SAXS and WAXS). Additionally, the influence of PTMO segment on the tensile properties and thermal and thermo-oxidative stability has been analysed.

\section{Experimental section}

\subsection{Synthesis of PETG-block-PTMO copolymers}

PETG-block-PTMO copolymers were prepared with several different PETG/PTMO weight ratios from dimethyl terephthalate (DMT, Sigma Aldrich), 1,2-ethylene glycol (ED, Sigma Aldrich), 1,4-cyclohexanedimethanol (CHDM, Sigma Aldrich) and poly(tetramethylene oxide) glycol (PTMG, Terathane 1000, DuPont, USA) with molecular weight of $1000 \mathrm{~g} \mathrm{~mol}^{-1}$, in two steps: the first one involved the transesterification of DMT by ED and CHDM in the presence of zinc acetate $\left(\mathrm{Zn}\left(\mathrm{CH}_{3} \mathrm{COO}\right)_{2}\right.$, Sigma Aldrich); whereas the second step was polycondensation, where PTMG was added in the presence of germanium dioxide $\left(\mathrm{GeO}_{2}, \mathrm{PPM}\right.$ Pure Metals $\left.\mathrm{GmbH}\right)$ and thermal stabilizer Irganox 1010 (Ciba - Geigy, Switzerland). The reaction was carried out in the $1 \mathrm{dm}^{3}$ high pressure reactor (Autoclave Engineers Pennsylvania, USA) equipped with a vacuum pump, condenser, and cold trap for collecting the by-products. In the first step the reactor was charged with DMT, ED and CHDM with the proper ratios, and catalyst. The molar ratio of the diester (DMT) and diol (ED) was $1: 1.5$. The transesterification reaction, was carried out under a constant flow of nitrogen at temperature of 160-180 ${ }^{\circ} \mathrm{C}$ for $\mathrm{ca}$. two hours. During this step, methanol was distilled and collected as a by-product. The conversion of the transesterification reaction was calculated by monitoring the amount of effluent by-product. When the distillation of methanol has been ceasing, the reaction was completed and the temperature was gradually increased to up $210{ }^{\circ} \mathrm{C}$. Then, the PTMG and thermal stabilizer (Irganox 1010) along with the second catalyst were introduced to the reactor. The reaction temperature was increased to $250{ }^{\circ} \mathrm{C}$. Vacuum was applied gradually and the final pressure was lower than $25 \mathrm{~Pa}$. The stirring torque change was monitored in order to estimate the melt viscosity of the product at temperature of $250{ }^{\circ} \mathrm{C}$. The process was found to be finished, when the reaction mixture reached the same value of melt viscosity, which was estimated by monitoring the stirring torque. Finally the material was extruded from the reactor using compressed nitrogen into water bath.

The polycondensation reaction time was 2-3.5 h dependent from the weight content of PTMO segments. The PETG copolymer was synthesized following the same procedure. The reactions involved within this process are formulated in Scheme 1.

\subsection{Samples preparation}

The dumbbell shape samples (type A3) for DMTA and tensile measurements were obtained by injection moulding using Boy 15 (Dr BOY GmbH \& Co., Germany) injection moulding machine with the following parameters: injection pressure $55 \mathrm{MPa}$, melt temperature $225{ }^{\circ} \mathrm{C}$, mold temperature $30{ }^{\circ} \mathrm{C}$, holding down pressure of $20 \mathrm{MPa}$ for $15 \mathrm{~s}$ and cooling time of $10 \mathrm{~s}$.

Thin polymer foils with the thickness of $\sim 220 \mu \mathrm{m}$ were prepared for SAXS and WAXS measurements by press moulding 


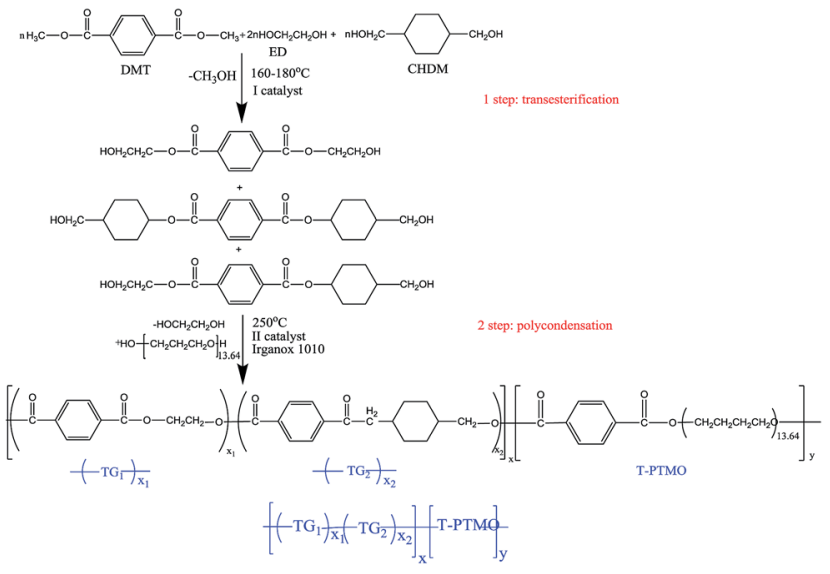

Scheme 1 Synthesis of PETG-block-PTMO copolymers.

(Collin P 200E) at $230^{\circ} \mathrm{C}$, and the pressure of 5 bar for $2 \mathrm{~min}$ and $10 \mathrm{bar}$ for another $1 \mathrm{~min}$. The thickness of thin films was measured with a Micrometer mod. 293-521 from Mitutoyo. The thickness is an average value.

\subsection{Characterization methods}

All the NMR spectra were collected at $25{ }^{\circ} \mathrm{C}$ on an Agilent DirectDrive II $600 \mathrm{M} \mathrm{Hz}$ spectrometer equipped with four broad-band rf channels and a $5 \mathrm{~mm}{ }^{1} \mathrm{H}^{19}{ }^{19} /{ }^{15} \mathrm{~N}^{-31} \mathrm{P}$ pulse field gradient (PFG) probe. Samples were dissolved in $d$-chloroform at the concentration of $10 \mathrm{mg} \mathrm{mL}{ }^{-1}$. During the acquisition periods, ${ }^{13} \mathrm{C}$ was decoupled by WURST-40. The ${ }^{1} \mathrm{H}$ quantitative NMR spectra were collected with a $1.7 \mathrm{~s}$ acquisition time, $20 \mathrm{~s}$ relaxation delay, $9.6 \mathrm{kHz}$ spectral window, 32 transients, and $9.1 \mu \mathrm{s}$ $90^{\circ}$ pulse width.

The PETG-block-PTMO copolymers were characterized using an FTIR spectrophotometer (Bruker Optik GmbH model Tensor 27). Measurements were carried out using the attenuated total reflectance (ATR) technique. Each sample was scanned 32 times at the resolution of $2 \mathrm{~cm}^{-1}$ over the frequency range of 4000$400 \mathrm{~cm}^{-1}$.

The intrinsic viscosity $[\eta]$ of the series of block copolymers was determined at $30{ }^{\circ} \mathrm{C}$ in the mixture of phenol/1,1,2,2tetrachloroethane (60/40 by weight). The concentration of the polymer solution was of $0.5 \mathrm{~g} \mathrm{dl}^{-1}$. The measurement was carried using a capillary Ubbelohde viscometer (type Ic, $K=$ $0.03294)$.

Size exclusion chromatography (SEC) in 1,1,1,3,3,3-hexafluoroisopropanol (HFIP) was performed at $40{ }^{\circ} \mathrm{C}$ on a system equipped with a waters 1515 Isocratic HPLC pump, a waters 2414 refractive index detector $\left(35^{\circ} \mathrm{C}\right)$, a waters 2707 auto sampler, and a PSS PFG guard column followed by two PFG-linear-XL $(7 \mu \mathrm{m}, 8 \times 300 \mathrm{~mm})$ columns in series. HFIP with potassium trifluoroacetate $\left(3 \mathrm{~g} \mathrm{~L}^{-1}\right)$ was used as the eluent at a flow rate of $0.8 \mathrm{~mL} \min ^{-1}$. The molecular weights were calculated against poly(methyl methacrylate) standards (Polymer Laboratories, $M_{\mathrm{p}}=580 \mathrm{Da}$ up to $\left.M_{\mathrm{p}}=7.1 \times 106 \mathrm{Da}\right)$.

The amorphous structure of the samples was confirmed by differential scanning calorimeter (DSC). Measurements were carried out with a DSC1 (Mettler Toledo) which was calibrated for the temperature and melting enthalpy by using indium and $n$-octane as a standards under a $\mathrm{N}_{2}$ atmosphere with a heating rate of $10 \mathrm{~K} \mathrm{~min}^{-1}$ in the temperature range of $25-300{ }^{\circ} \mathrm{C}$. Then, from the second heating the glass transition $T_{\mathrm{g}}$ and corresponding heat capacity were determined.

The dynamic mechanical thermal analysis (DMTA) has been performed using a Polymer Laboratories MK II apparatus working in a bending mode in a temperature range from $-100{ }^{\circ} \mathrm{C}$ to the polymer melt temperature, at a frequency of $1 \mathrm{~Hz}$ and the heating rate of $3{ }^{\circ} \mathrm{C} \mathrm{min}^{-1}$. The properties were determined on the basis of modulus changes and the ability of attenuation as a function of temperature and frequency of load changes.

WAXS and SAXS experiments were carried out at the NCD beamline (BL11) of the Alba synchrotron radiation light facility of Cerdanyola del Vallè s (Catalunya). The beam was monochromatized to a wavelength of $0.1 \mathrm{~nm}$. The SAXS detector was calibrated with diffractions of a silver behenate standard sample. The diffraction profiles were normalized to the beam intensity and corrected considering the empty sample background. The X-ray scattering intensity, $I(q)$, was experimentally determined as a function of the scattering vector, which was obtained according to equation: $q=(4 \pi / \lambda) \sin \theta$, where $\lambda$ is the $\mathrm{X}$-ray wavelength and $2 \theta$ is the scattering angle. The position of the scattering maximum, on the Lorentz-corrected SAXS patterns, was used for the calculation of the long period $(L)$ according to eqn (2): $L=2 \pi / q_{\max }$.

The tensile properties of the prepared PETG-block-PTMO copolymers were measured using Autograph AG-X plus (Shimadzu) tensile testing machine equipped with a $1 \mathrm{kN}$ Shimadzu load cell, an contact optical long travel extensometer and the TRAPEZIUM X computer software, operated at a constant crosshead speed of $5 \mathrm{~mm} \mathrm{~min}^{-1}$. Measurements were performed at room temperature on the dumbbell samples with the grip distance of $20 \mathrm{~mm}$. According to DIN 53455 standard, the tensile modulus, stress at $100 \%$ strain, yield stress and strain, stress and elongation at break of the block copolymers were determined. Five measurements were conducted for each sample, and the results were averaged to obtain a mean value.

The thermo-oxidative and thermal stability of the synthesised PETG-block-PTMO copolymers were evaluated by thermogravimetry (TGA 92-16.18 Setaram) using the system measuring simultaneously TG-DSC. Measurements were carried out in an oxidizing atmosphere i.e. dry, synthetic air $\left(\mathrm{N}_{2}: \mathrm{O}_{2}=\right.$ $80: 20 \mathrm{vol} \%$ ) and in an inert atmosphere (argon). The study was conducted at a heating rate of $10{ }^{\circ} \mathrm{C} \mathrm{min}^{-1}$ in the temperature range of $20-700{ }^{\circ} \mathrm{C}$. Measurements were conducted in accordance with the PN-EN ISO 11358:2004 standard.

\section{Results and discussion}

\subsection{NMR and FTIR analyses on structure and composition}

PETG-block-PTMO samples were characterized by an improved PETG-block-PTMO samples were characterized by an improved quantitative NMR technique to verify the structure details via end-groups estimation method ${ }^{34}$ following the similar 
procedure used for the characterization of poly(ether ester) multiblock copolymers. ${ }^{35,36}$ Fig. 1 shows the ${ }^{1} \mathrm{H}$ NMR spectra of PETG-block-PTMO 95/5 copolymer, with the interested region enlarged, while others are presented in ESI (Fig. S1-S3†). It is hard to assign these fine peaks to corresponding chemical structure, thus the ${ }^{1} \mathrm{H}$ NMR, ${ }^{1} \mathrm{H}^{-13} \mathrm{C}$ gHSQC and ${ }^{1} \mathrm{H}^{-1} \mathrm{H}$ gCOSY 2D NMR spectra of PETG copolymer (copolymer of PET and PCT with PCT ratio of 30\%) were carried for comparison (Fig. 2, S4 and $\mathrm{S} 5 \dagger$ ). The methylene (yellow coloured cross-peaks) and methine groups (blue coloured cross-peaks) are distinguished by ${ }^{1} \mathrm{H}^{13} \mathrm{C}$ gHSQC spectra of PETG homopolymers (Fig. S5 $\dagger$ ).

In ${ }^{1} \mathrm{H}$ NMR, the peaks at chemical shifts of $1.80 \mathrm{ppm}$ (f2) and $2.05 \mathrm{ppm}$ (f1) are assigned to the methine groups, while that at 4.17 (e2) ppm and $4.29 \mathrm{ppm}$ (e1) to the methylene groups connected with ester bonds at trans- and cis-form of cyclohexanedimethylene repeating units, respectively. This is confirmed by the ${ }^{1} \mathrm{H}^{-1} \mathrm{H}$ gCOSY $2 \mathrm{D}$ NMR spectra of PETG homopolymers, as presented in Fig. 2, where the correlations between $\mathrm{e} 2$ and $\mathrm{f} 2$, e 1 and $\mathrm{f} 1$, are observed, confirming they are adjacent groups. The correlation between peak a and $b$ indicates these two methylene groups are chain-end groups, which can be used for the estimation of polymer structure.

The number-average molecular weights $\left(M_{\mathrm{n}}\right)$ of PETG-blockPTMO copolyesters, in addition to PET, PTMO, PCT content and average repeating units in a polymer chain, can be estimated from the hydroxyl methylene chain ends (peak a), the methylene groups from PET segments (peak d), from PCT segments connected with ester bonds (peak e), from PTMO segments linked to ether or ester bonds (peak i + peak k), respectively, via the following equations:

$$
\begin{gathered}
\frac{I_{\mathrm{a}}}{I_{\mathrm{d}}}=\frac{4}{4 N_{\mathrm{ET}}} \\
\frac{I_{\mathrm{a}}}{I_{\mathrm{c}}}=\frac{4}{4 N_{\mathrm{CT}}} \\
\frac{I_{\mathrm{a}}}{I_{\mathrm{i}}+I_{\mathrm{k}}}=\frac{4}{4 N_{\mathrm{TMO}}} \% \\
M_{\mathrm{n}}=192 N_{\mathrm{ET}}+274 N_{\mathrm{CT}}+72 N_{\mathrm{TMO}}
\end{gathered}
$$

where $I_{\mathrm{a}}, I_{\mathrm{d}}, I_{\mathrm{e}}, I_{\mathrm{i}}, I_{\mathrm{k}}$ is the integrals from corresponding peaks, $N_{\mathrm{ET}}, N_{\mathrm{CT}}, N_{\mathrm{TMO}}$ the average repeating units of ET, CT and TMO
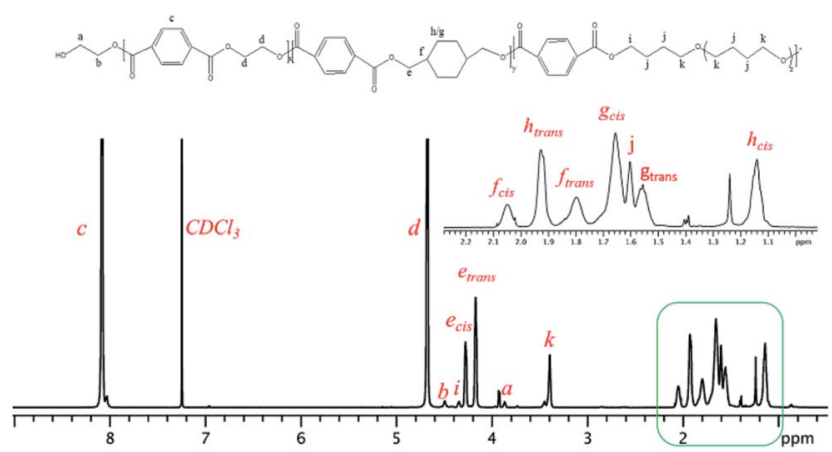

Fig. $1{ }^{1} \mathrm{H}$ quantitative NMR spectra of PETG-block-PTMO 95/5 sample. Solvent: $\mathrm{CDCl}_{3}$.

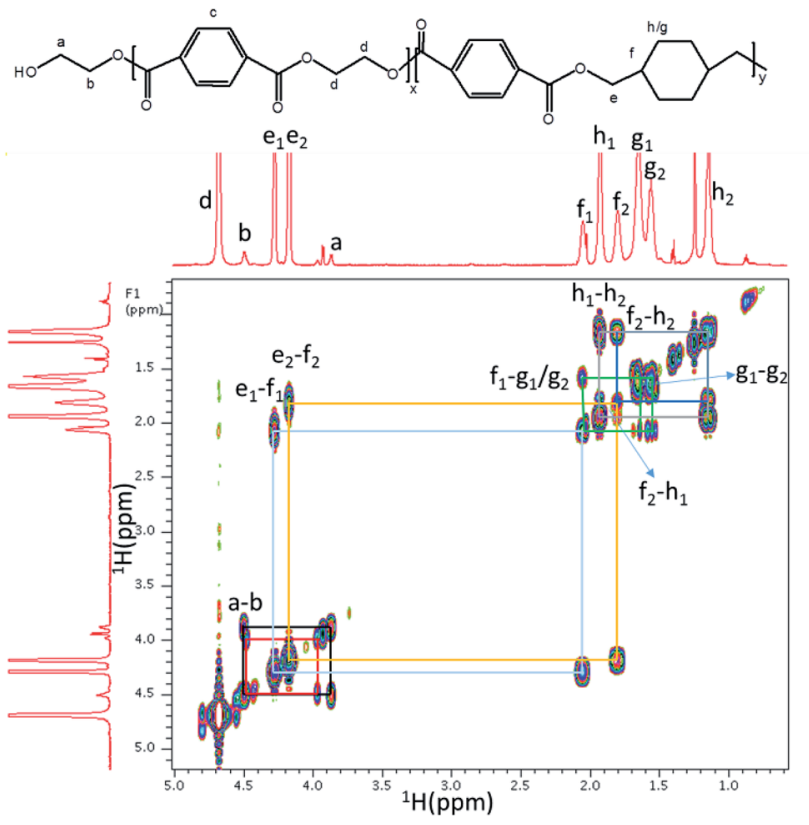

Fig. $2{ }^{1} \mathrm{H}-{ }^{1} \mathrm{H}$ gCOSY NMR spectrum of PETG sample. Solvent: $\mathrm{CDCl}_{3}$.

in PETG-block-PTMO chain, respectively. The calculated data from NMR spectra are listed in Table 1.

The number-average molecular weights of PETG-block-PTMO copolymers estimated from ${ }^{1} \mathrm{H}$ NMR spectra are all around $20 \mathrm{~kg} \mathrm{~mol}{ }^{-1}$, indicating the well control of the molecular weights during polymerization. The average mole ratio of the repeating units of ET to CT $\left(r_{\mathrm{ET} / \mathrm{CT}}\right)$ in the copolymers, is around 2. This is slightly lower than the feeding ratio (2.33), but is quite reasonable since some ethylene glycol is distilled out during polymerization process. The content of PTMO in the poly(ether ester)s (weight fraction, PTMO\%), is lower than the feeding ratio. This means some of the PTMO decomposed during the polymerization process, which is common during the traditional poly(ether ester) synthesis process, leading to the decrement of PTMO content in the final product. On the other hand, the trans/cis ratio of CHDM in PETG-block-PTMO, calculated from the integral ratio from peak e2 to e1, is all about 1.50, similar to previous PETG copolyester systems (1.45).

FTIR analysis. Fig. 3a shows the FTIR spectra of PET and PETG, in which the peak at $1712 \mathrm{~cm}^{-1}$ displays the $\mathrm{C}=\mathrm{O}$ of ester groups, and the $\mathrm{C}-\mathrm{H}$ out-of-plane deformation of two carbonyl substituents on the aromatic ring depicts at $730 \mathrm{~cm}^{-1} \cdot{ }^{\mathbf{1 4 , 3 7}}$ The two peaks at 1410 and $1240 \mathrm{~cm}^{-1}$ are ascribed to $-\mathrm{CH}_{2}-$ deformation band and $\mathrm{C}(\mathrm{O})-\mathrm{O}$ stretching of ester groups, respectively. ${ }^{38}$ The asymmetric and symmetric aliphatic $\mathrm{C}-\mathrm{H}$ stretching vibrations were detected in 2907 and $2968 \mathrm{~cm}^{-1}$ for PET. ${ }^{39}$ However, in PETG copolymer, the peaks attributed to $\mathrm{C}-\mathrm{H}$ stretching were shifted to the lower wavenumbers at 2852 and $2924 \mathrm{~cm}^{-1}$ which is due to the presence of methyl groups in the structure of this polymer. ${ }^{31,40}$ Moreover, the $\mathrm{C}-\mathrm{H}$ stretching peak of cyclohexylene ring was found at $958 \mathrm{~cm}^{-1}$. Fig. $3 \mathrm{~b}$ depicts the FTIR spectra of PETG-block-PTMO copolymers with various content of PTMO ranging from $0-30 \mathrm{wt} \%$. It is obvious that no 
Table 1 The structure of PETG-block-PTMO copolymers from ${ }^{1} \mathrm{H}$ NMR spectra

\begin{tabular}{|c|c|c|c|c|c|c|}
\hline Sample & $N_{\mathrm{ET}}$ & $N_{\mathrm{CT}}$ & $N_{\text {TMO }}$ & $M_{\mathrm{n}}\left(\mathrm{kg} \mathrm{mol}^{-1}\right)$ & $r_{\mathrm{ET} / \mathrm{CT}}$ & РТMO \% \\
\hline PETG-block-PTMO 95/5 & 59.1 & 28.1 & 10.5 & 19.8 & 2.10 & 3.8 \\
\hline PETG-block-PTMO 90/10 & 69.4 & 31.7 & 21.0 & 23.5 & 2.18 & 6.4 \\
\hline PETG-block-PTMO 70/30 & 58.1 & 29.3 & 81.2 & 25.0 & 1.99 & 23.4 \\
\hline
\end{tabular}

new peaks and bonds were observed in the spectra of PEEs rather than PETG copolymers. Only a small shift in PETG spectra from $1244 \mathrm{~cm}^{-1}$ to lower wavenumbers was observed with the increase in PTMO content. Moreover, at higher content of PTMO soft segments (30\%), the increase in the magnitude of the absorbance (relative intensity) was observed because of the flexibility of PEEs. In this case, the intensity of carbonyl stretching peaks at $1244 \mathrm{~cm}^{-1}$ and $1716 \mathrm{~cm}^{-1}$ increased significantly.

\subsection{Physicochemical properties of PETG-block-PTMO copolymers}

A series of thermoplastic copoly(ester-ethers) based on rigid PETG and flexible PTMO segments (PETG-block-PTMO) was
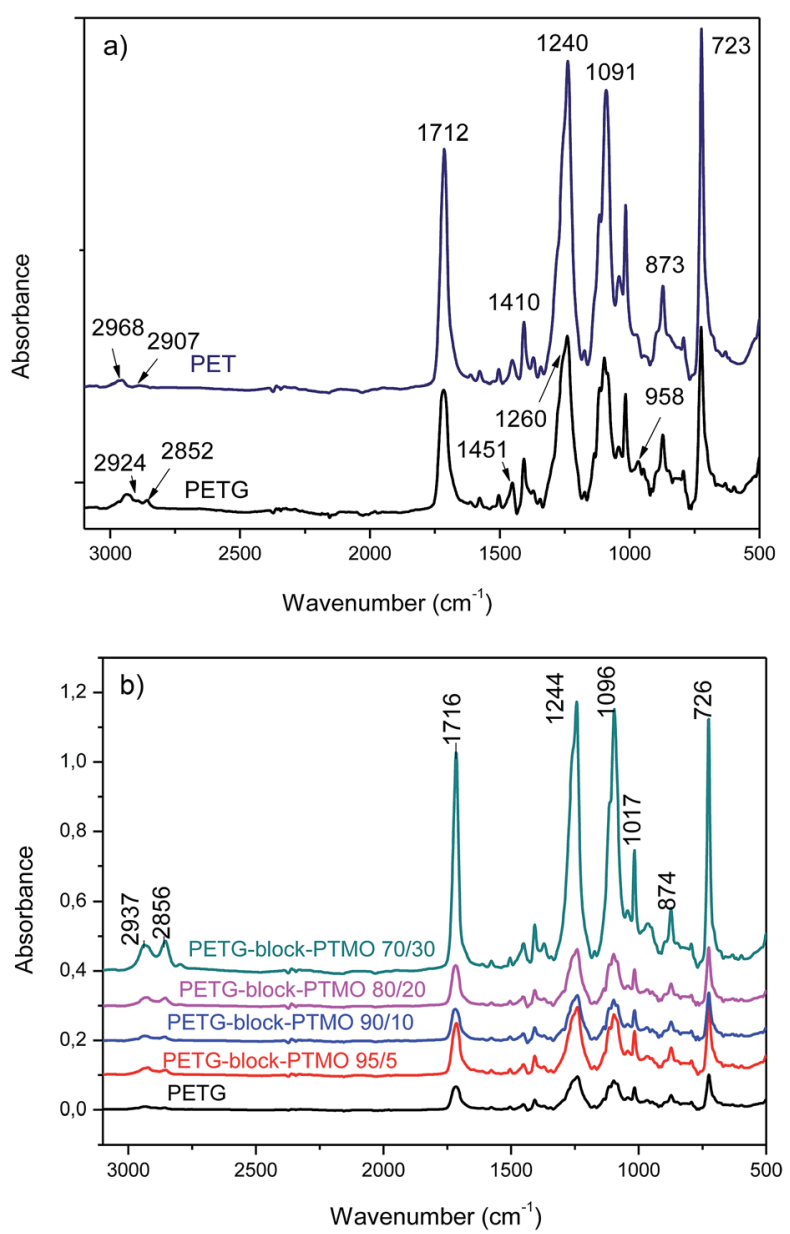

Fig. 3 FTIR spectra of (a) PET and PETG (b) PETG-block-PTMO copolymers and PETG. prepared by two-stages reaction, by means of transesterification and polycondensation. Since, the PETG-block-PTMO copolymer can be presented as a random copolyester (terephthalate units ( $\mathrm{TG}_{1}$ and $\mathrm{TG}_{2}$ ) and T-PTMO units), one can find the possibility to calculate the degree of polymerization (polycondensation) of rigid segments. The theoretical chemical composition of the series of synthesised block copolymers and its basic physicochemical properties, by means of number-average molecular mass $\left(M_{\mathrm{n}}\right)$, weight-average molecular mass $\left(M_{\mathrm{w}}\right)$, polydispersity index (PDI), intrinsic viscosity [ $\eta$ ], glass transition temperature $\left(T_{\mathrm{g}}\right)$ and corresponding heat capacity are summarized in Table 2. The average length of the rigid PETG segment (degree of polymerization " $x$ ") was calculated accordingly to $1 \mathrm{~mol}$ of flexible segment T-PTMO $(y=1$ on Scheme 1). The degree of polymerization of PETG rigid segments, calculated from the reaction compositions, ranged from 111.82 (PETG-block-PTMO 95/5) to 13.72 (PETG-block-PTMO 70/30). The limiting viscosity number $[\eta]$ of polymers/copolymers can be influenced by the molecular chains' weight and their flexibility. ${ }^{41}$ In the present study the value of $[\eta]$ for PETG homopolymer equals to 0.696, which was slightly higher than that for the previously published in. ${ }^{42}$ While, the values of intrinsic viscosity for the series of copolymers increase along with an increase of weight fraction of PTMO segments from $0.59 \mathrm{dl} \mathrm{g}^{-1}$ to $0.659 \mathrm{dl} \mathrm{g}^{-1}$ (Table 2). The increase of $[\eta]$ values along with an increase in the PTMO content in the copolymer chain can suggest that the obtained block copolymers exhibit comparable or higher molecular weight than PETG, synthesized at the same conditions. This suggestion was confirmed by SEC analysis. The number of average molecular masses of the copolyesters in these series ranged from 19512 to $27086 \mathrm{~g} \mathrm{~mol}^{-1}$ (Table 2), while the polydispersity index was in the range $2.13-2.48$. At the same time, from the DSC thermograms (Fig. 4) one can find that PETG and PTMO segments did not segregate into separate phase in the solid state (only one $T_{\mathrm{g}}$ is observed), which indicate on the thermodynamic miscibility of the segments. The $T_{\mathrm{g}}$ value for PTMO equals to $-84{ }^{\circ} \mathrm{C},{ }^{2,43}$ whilst the glass transition temperature for PETG is $79.8^{\circ} \mathrm{C}$ (Table 2), and since the values of $T_{\mathrm{g}}$ (only one glass transition shift is observed for all systems) decrease along with an increasing content of PTMO, one can draw the conclusions about the miscibility of both phases.

\subsection{Phase structures and morphology}

The results of dynamic mechanical thermal analysis for the series of PETG-block-PTMO are presented in Fig. 5, where the storage and loss modulus (a) and $\tan \delta$ (b) are plotted as a function of temperature. For PETG-block-PTMO copolymers 
Table 2 Composition and basic physicochemical characteristics of PETG and PETG-block-PTMO copolymers ${ }^{a}$

\begin{tabular}{|c|c|c|c|c|c|c|c|c|}
\hline Sample & $w_{\mathrm{s}}(\mathrm{wt} \%)$ & $x\left(\mathrm{~mol} \mathrm{~mol}^{-1}\right)$ & {$[\eta]\left(\mathrm{dl} \mathrm{g}^{-1}\right)$} & $M_{\mathrm{n}}\left[\mathrm{g} \mathrm{mol}^{-1}\right]$ & $M_{\mathrm{w}}\left[\mathrm{g} \mathrm{mol}^{-1}\right]$ & DPI & $T_{\mathrm{g}}\left({ }^{\circ} \mathrm{C}\right)$ & $\Delta c_{\mathrm{p}}\left(\mathrm{J} \mathrm{gK}^{-1}\right)$ \\
\hline PETG & 0 & - & 0.696 & 19512 & 43125 & 2.21 & 79.8 & 0.380 \\
\hline PETG-block-PTMO 95/5 & 5 & 111.82 & 0.590 & 20190 & 44399 & 2.20 & 67.8 & 0.330 \\
\hline PETG-block-PTMO 80/20 & 20 & 23.54 & 0.620 & 27086 & 67173 & 2.48 & 46.7 & 0.547 \\
\hline PETG-block-PTMO 70/30 & 30 & 13.72 & 0.659 & 26510 & 59647 & 2.25 & 17.4 & 0.416 \\
\hline
\end{tabular}

${ }^{a} w_{\mathrm{s}}$-weight fraction of PTMO segments; $x$-degree of polymerization of rigid segment with reference of 1 mol of T-PTMO unit; [ $\eta$ ]-limiting viscosity number; $M_{\mathrm{n}}$-number-average molecular mass; $M_{\mathrm{w}}$-weight-average molecular mass; PDI-polydispersity index; $T_{\mathrm{g}}-\mathrm{glass}$ transition temperature; $\Delta c_{\mathrm{p}}$-heat capacity.

the $\alpha$-relaxations observed on the maximum of loss modulus (Fig. 5a) and $\tan \delta$ (Fig. 5b) is due to the glass transition of soft amorphous phase. The drop of the storage modulus ( $\left.E^{\prime}\right)$ (Fig. 5a) is dependent on composition of the series of block copolymers and is related to the glass transition of the synthesized material. A single drop, observed for each copolymer, as well as for neat PETG suggest on the miscibility of both phases, unlike in the case of polyester elastomers based on PET and PEO, where the presence of four different domains dependent on thermal history and composition have been observed by Fakirov et al. ${ }^{44}$ For neat PETG one can observe two dynamic mechanical viscoelastic relaxations $\alpha$ and $\beta$. The $\sim 80^{\circ} \mathrm{C} \alpha$-relaxation on $\tan \delta$ curves is the glass transition. The $\beta$-relaxation in the temperature range of -80 to $-30{ }^{\circ} \mathrm{C}$ on loss modulus $\left(E^{\prime}\right)$ and $\tan \delta$ curves is attributed to the re-orientation of the hydroxyl groups and local motions of the carboxyl groups in the amorphous phase. ${ }^{45}$ As the weight content of flexible segments in PETG-block-PTMO copolymers increased, the glass transition peak $(\alpha)$ of soft phase is shifted toward lower temperature, from $81{ }^{\circ} \mathrm{C}$ (PETG) to $42{ }^{\circ} \mathrm{C}$ (for PETG-block-PTMO 70/30). The DSC and DMTA analyses confirmed the two-phase structure of PETGblock-PTMO copolymers and thermodynamic miscibility of both phases.

The morphology of PETG and PETG block copolymers films was studied by SAXS and WAXS techniques. Fig. 6 and 7 show the SAXS and WAXS patterns of PETG and PETG based block

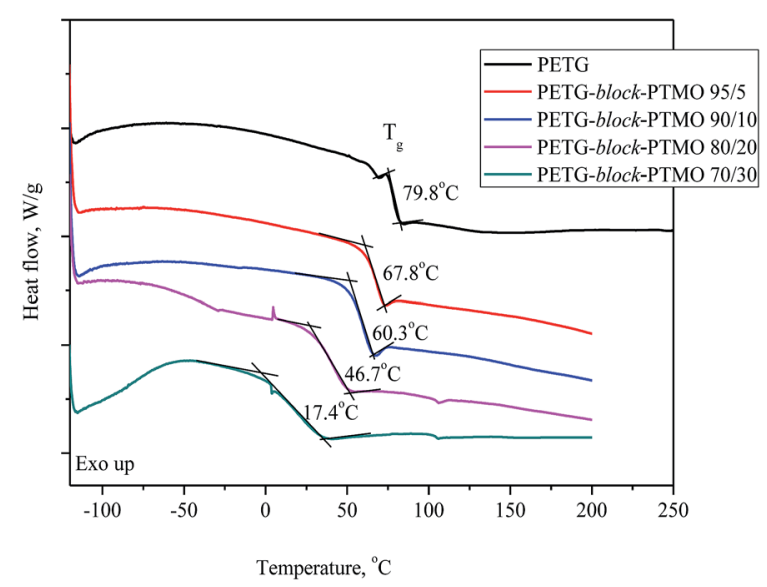

Fig. 4 DSC thermograms of PETG-block-PTMO copolymers at different wt\% content of PTMO segments. copolymers in comparison with semicrystalline PET film. For PETG and PETG copolymers containing above $10 \mathrm{wt} \%$ of PTMO blocks in copolymer the lamellar nanostructure formation was confirmed. PETG copolymers exhibit the same diffraction patterns and positions as PET, indicating that PET crystals are present in annealed films. The determined values of long period for PETG and PETG based block copolymers are presented in Table 3. The PETG-block-PTMO copolymers show higher values of long period in comparison with semi-crystalline PET and PETG. The higher values of long period for PETG copolymers can be attributed to the growth of the amorphous domains and
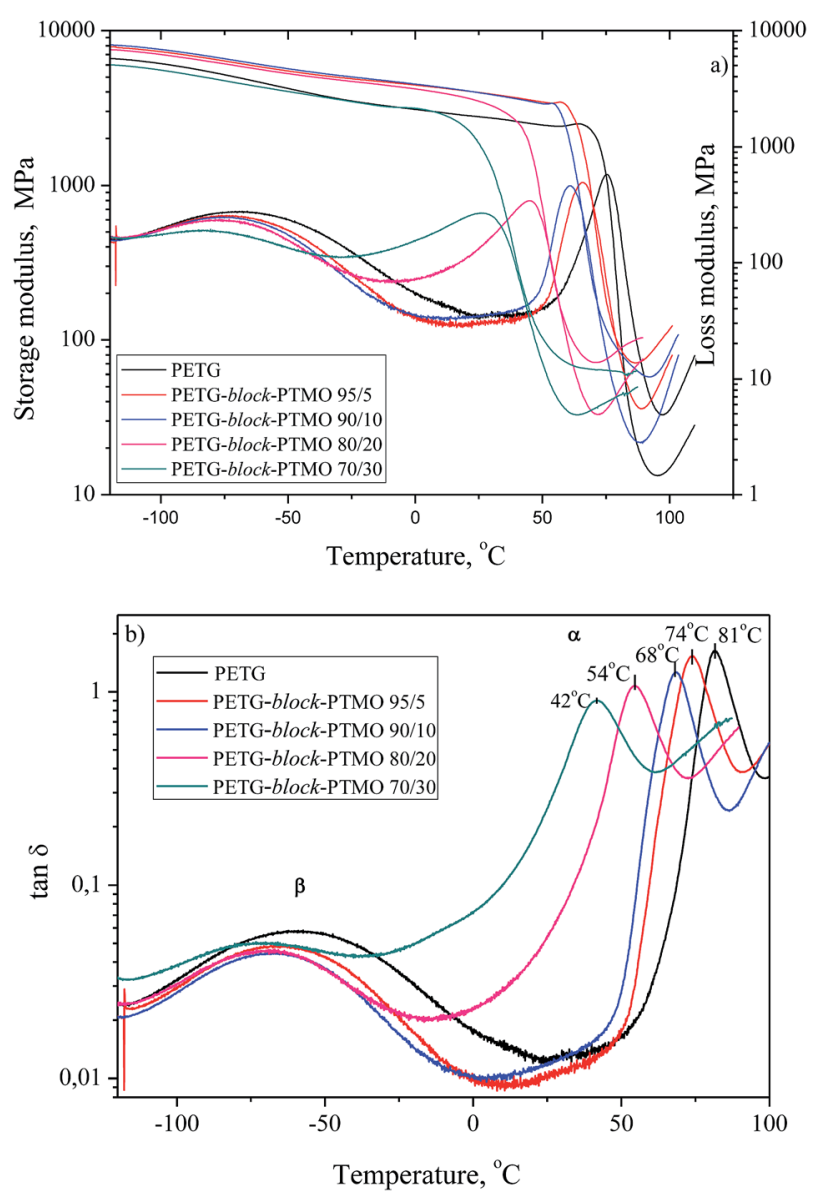

Fig. 5 The storage modulus $E^{\prime}$, loss modulus $E^{\prime}$ and $\tan \delta$ as a function of temperature for PETG-block-PTMO copolymers. 


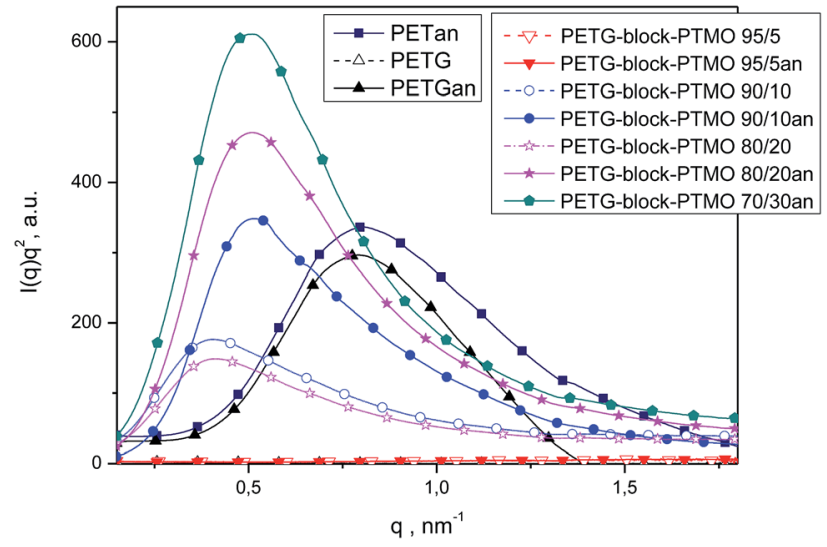

Fig. 6 Lorentz-corrected SAXS patterns for prepared amorphous and annealed above $T_{\mathrm{g}}$ films of PETG, PETG copolymers and semicrystalline PET.

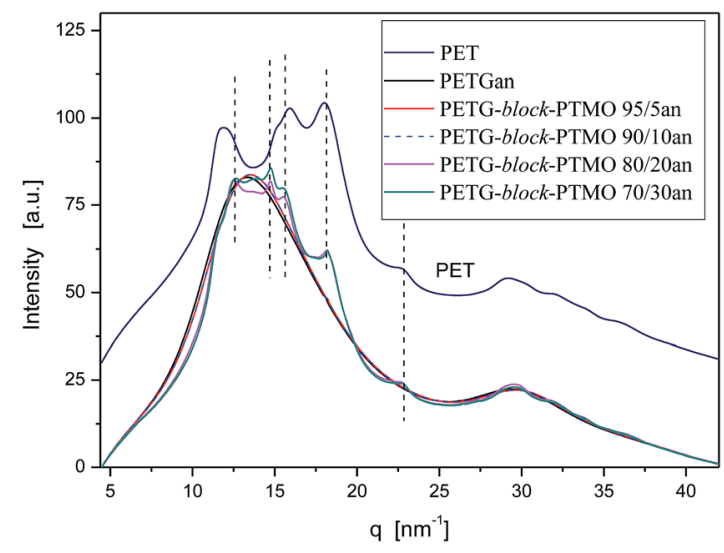

Fig. 7 WAXS patterns for annealed films of PETG, PETG copolymers and semicrystalline PET.

sub-domains resulting in the coalescence of PETG and PTMO sub-domains. ${ }^{41}$

\subsection{Tensile properties}

In block copolymers, the nanometric structure of rigid and flexible segments is responsible for their mechanical properties by means of: rigid segments of PETG for an increase in strength, whereas flexible segments of PTMO for the elastic properties. Stress-strain curves of the synthesised block copolymers are presented in Fig. 8. Moreover, the characteristic parameters, i.e. values of Young's modulus $(E)$, tensile strength and elongation at yield $\left(\sigma_{\mathrm{y}}, \varepsilon_{\mathrm{y}}\right)$ and strength and elongation at break $\left(\sigma_{\mathrm{b}}, \varepsilon_{\mathrm{b}}\right)$ are
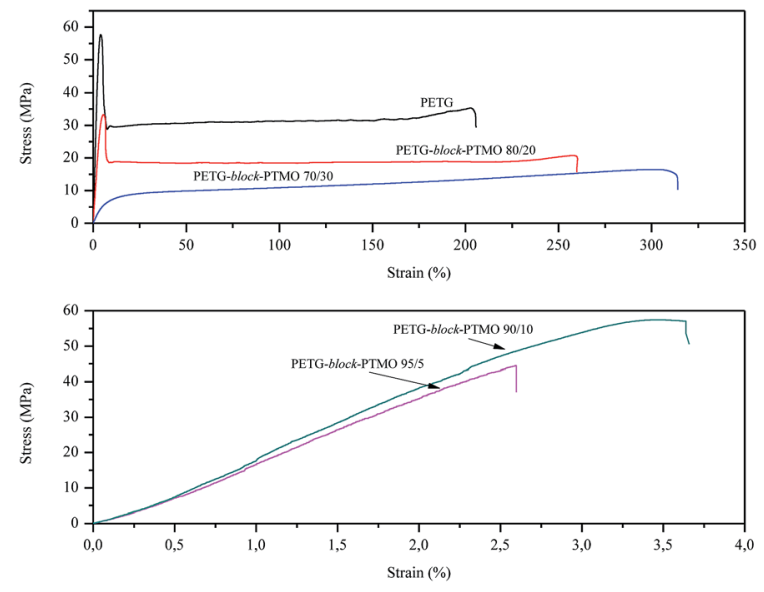

Fig. 8 The stress-strain curves of PETG-block-PTMO copolymers.

summarized in Table 4. Along with an increase in flexible segments content, the decrease in Young's modulus and strength at break were observed, which results from the decrease of domains that are contributed to strength. The tensile strength at yield decreased from 57.7 MPa (for PETG) to 44.5 MPa for PETG-block-PTMO 95/5 (only for these two samples it could have been estimated), whereas the values of elongation at break for the series of block copolymers, firstly decreased of about two orders of magnitude from 205.9\% (PETG) to ca. 3\% (for PETG-block-PTMO 95/5 and PETG-block-PTMO 90/10) and then increase to $314.1 \%$ for PETG-block-PTMO 70/30. This initial decline and further increase (above $20 \mathrm{wt} \%$ of PTMO) in the values of strength and elongation at break might result from the thermodynamic miscibility of both phases, which was previously confirmed by DSC and DMTA analyses. Moreover, following Brostow et al., ${ }^{\mathbf{4 6}, 47}$ who demonstrated the existence of a quantitative relationship between toughness $(\tau)$ and brittleness $(B)$, valid for polymers with a wide range of chemical structures and properties, one calculated $B$ accordingly to eqn (5):

$$
B=\frac{1}{\varepsilon_{\mathrm{b}} E^{\prime}}
$$

where $\varepsilon_{\mathrm{b}}$ is the elongation at break and $E^{\prime}$ is the storage modulus determined by DMTA at $1 \mathrm{~Hz}$ and $25^{\circ} \mathrm{C}$. The descriptor $B$ turned out to be useful in evaluating polymer blends, ${ }^{48}$ multi-layer laminate composites ${ }^{49}$ and many more. ${ }^{47}$ In the case of linear low density polyethylene/cycloolefin copolymer blends, ${ }^{\mathbf{4 8}}$ the rates of change of $\varepsilon_{\mathrm{b}}$ and $E^{\prime}$ were different, thus affecting the outcome in $B$ with changing concentration of components. $B$ decreased when a rigid copolymer was added to linear low-

Table 3 Long period determined for PETG and PETG copolymers films as prepared and later after annealing above $T_{\mathrm{g}}$

\begin{tabular}{|c|c|c|c|c|c|c|}
\hline Samples & PET & PETG & $\begin{array}{l}\text { PETG-block-PTMO } \\
95 / 5\end{array}$ & $\begin{array}{l}\text { PETG-block-PTMO } \\
90 / 10\end{array}$ & $\begin{array}{l}\text { PETG-block-PTMO } \\
80 / 20\end{array}$ & $\begin{array}{l}\text { PETG-block-PTMO } \\
70 / 30\end{array}$ \\
\hline Annealed films $L(\mathrm{~nm})$ & 7.79 & 7.93 & - & 12.21 & 12.31 & 12.42 \\
\hline
\end{tabular}


Table 4 Tensile properties of PETG-block-PTMO copolymers ${ }^{a}$

\begin{tabular}{llllrrr}
\hline Sample & $E(\mathrm{MPa})$ & $\sigma_{\mathrm{y}}(\mathrm{MPa})$ & $\varepsilon_{\mathrm{y}}(\%)$ & $\sigma_{\mathrm{b}}(\mathrm{MPa})$ & $\varepsilon_{\mathrm{b}}(\%)$ & \multicolumn{1}{c}{$B\left(10^{12}(\% \mathrm{~Pa})\right)$} \\
\hline PETG & $1470.0 \pm 20.9$ & $57.7 \pm 0.3$ & $3.9 \pm 0.2$ & $35.3 \pm 0.4$ & $205.9 \pm 2.9$ \\
PETG-block-PTMO 95/5 & $1425.9 \pm 43.3$ & - & - & $44.5 \pm 0.2$ & $2.6 \pm 0.2$ & 131.6806 \\
PETG-block-PTMO 90/10 & $1405.3 \pm 41.5$ & - & - & $57.4 \pm 0.9$ & $3.7 \pm 0.2$ & 92.7929 \\
PETG-block-PTMO 80/20 & $1072.1 \pm 31.2$ & $33.3 \pm 2.5$ & $5.5 \pm 0.1$ & $20.7 \pm 0.7$ & $259.7 \pm 9.8$ \\
PETG-block-PTMO 70/30 & $146.3 \pm 7.3$ & $3.4 \pm 0.2$ & $2.8 \pm 0.1$ & $16.5 \pm 0.8$ & $314.1 \pm 17.7$
\end{tabular}

${ }^{a} E$ - Young's Modulus (calculated from strain $0.05 \%$ to $0.25 \%$ ); $\sigma_{\mathrm{y}}$ - tensile strength at yield, $\sigma_{\mathrm{b}}, \varepsilon_{\mathrm{b}}-$ strength and elongation at break respectively, $B-\left(=1 /\left(\varepsilon_{\mathrm{b}} E^{\prime}\right)\right)-$ brittleness. ${ }^{46,47}$

density polyethylene. ${ }^{48}$ Herein, it can be seen (Table 4 ) that the addition of PTMO soft segments of 5 and $10 \mathrm{wt} \%$ increases the brittleness of the block copolymers. However, further increase in the amount of PTMO, i.e. 20 and $30 \mathrm{wt} \%$ reduces the brittleness of the materials.

\subsection{Thermal stability}

One of the parameters, that can limit the applications of the newly synthesised polymer materials, it's their thermal stability. The thermooxidative (air) and thermal (argon) stability of the PETG-block-PTMO copolymers were studied, and the TG and DTG curves with different contents of flexible PTMO segments are presented in Fig. 9 and 10. Additionally, the characteristic temperatures of 5, 25, 50 and 90\% of weight loss, in air and argon atmospheres are summarized in Table 5. In air atmosphere, the PETG exhibits two steps of degradation, which appear at $350-470{ }^{\circ} \mathrm{C}$ and $500-570{ }^{\circ} \mathrm{C}$. While, for PETG-block-
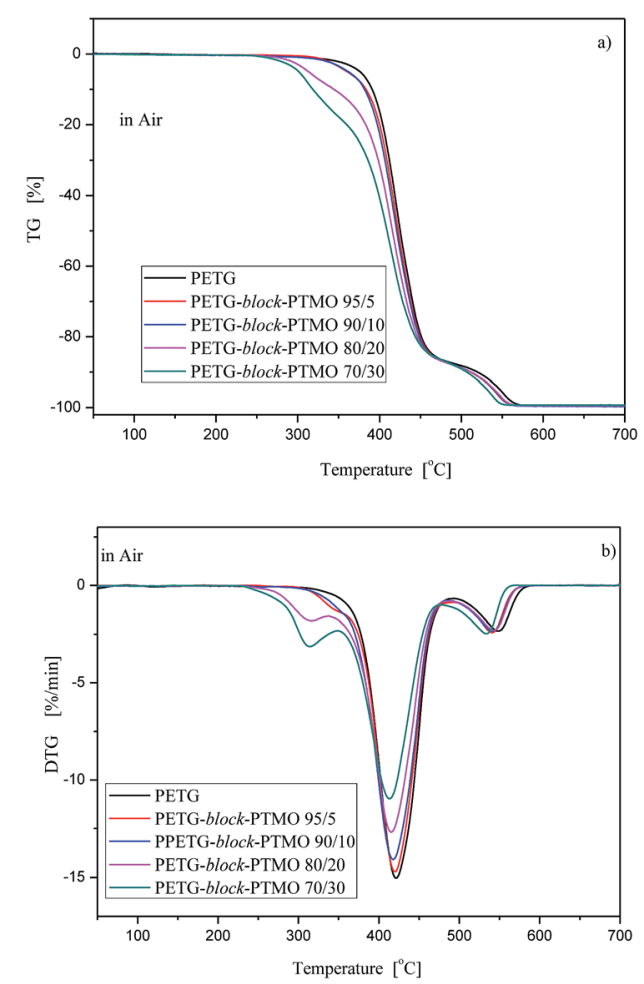

Fig. 9 TG (a) and DTG (b) curves for PETG-block-PTMO copolymers under air atmosphere at heating rate $10^{\circ} \mathrm{C} \mathrm{min}^{-1}$.
PTMO copolymers, three stages of the decomposition can be observed: (1) in the temperature range of $254-350{ }^{\circ} \mathrm{C}$; (2) in the temperature range $350-460{ }^{\circ} \mathrm{C}$ and (3) in the temperature range of $480-560{ }^{\circ} \mathrm{C}$. The first two stages one can attribute to the decomposition of flexible and rigid segments. In poly(etherester) block copolymers it is well-known that in raised temperature the attack of oxygen molecules is initiated in the flexible segment and, in most cases occurs at the $\alpha$-carbon atom to the ether oxygen atom. ${ }^{\mathbf{4 1 , 5 0}}$ In turn, the third stage is attributed to the decomposition of residue. Additionally, the values of weigh losses decrease along with the increasing content of PTMO flexible segments of about 70 to $16{ }^{\circ} \mathrm{C}$, for $5 \%$ and $90 \%$ weight loss, respectively.

Moreover, from the shapes of TG and TDG curves one can observe that in argon atmosphere, the thermal behaviours of PETG-block-PTMO copolymers were comparable to one another,
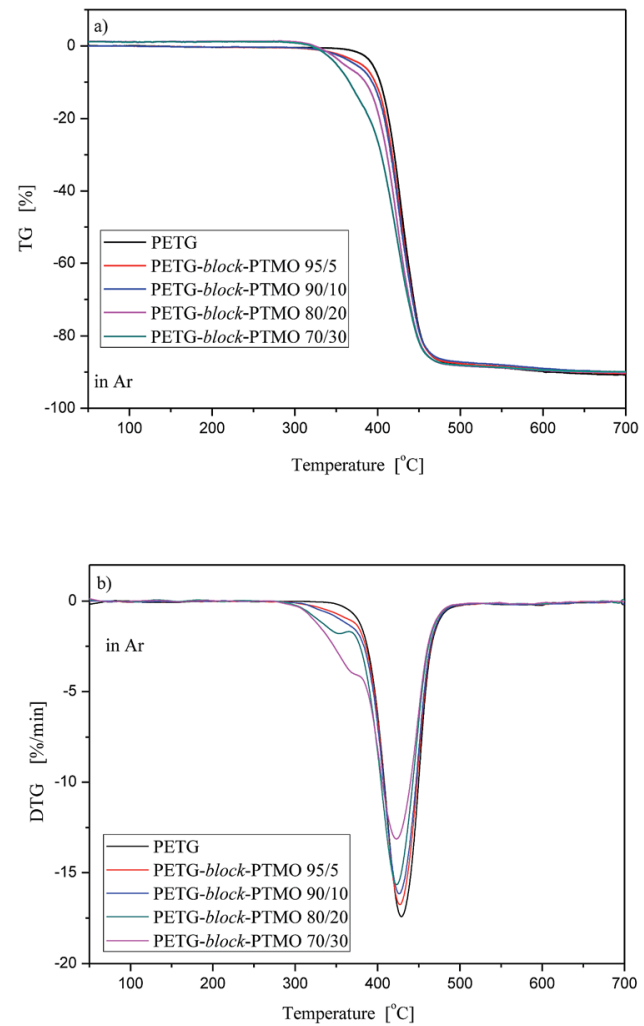

Fig. 10 TG (a) and DTG (b) curves for PETG-block-PTMO copolymers under argon atmosphere at heating rate $10^{\circ} \mathrm{C} \mathrm{min}^{-1}$. 
Table 5 TGA data: temperature of $5 \%, 25 \%, 50 \%$ and $90 \%$ weight loss in an inert and oxidizing atmosphere

\begin{tabular}{|c|c|c|c|c|c|c|c|c|}
\hline \multirow[b]{2}{*}{ Sample } & \multicolumn{2}{|c|}{$T_{5 \%},{ }^{\circ} \mathrm{C}$} & \multicolumn{2}{|c|}{$T_{25 \%},{ }^{\circ} \mathrm{C}$} & \multicolumn{2}{|c|}{$T_{50 \%},{ }^{\circ} \mathrm{C}$} & \multicolumn{2}{|c|}{$T_{90 \%},{ }^{\circ} \mathrm{C}$} \\
\hline & $\mathrm{Ar}$ & Air & $\mathrm{Ar}$ & Air & $\mathrm{Ar}$ & Air & $\mathrm{Ar}$ & Air \\
\hline PETG-block-PTMO 95/5 & 379.8 & 360.5 & 414.2 & 405.1 & 429.6 & 422.9 & 637.3 & 511.4 \\
\hline PETG-block-PTMO 90/10 & 372.5 & 361.9 & 412.6 & 402.5 & 428.6 & 421.1 & 702.5 & 512.1 \\
\hline PETG-block-PTMO 80/20 & 358.9 & 313.2 & 407.4 & 392.2 & 424.5 & 415.8 & 664.7 & 512.1 \\
\hline
\end{tabular}

and only in lower temperature range $\left(280-390{ }^{\circ} \mathrm{C}\right)$ the differences were observed. It was strongly connected with the PTMO flexible segment content. Along with an increase in of PTMO content the temperature at the maximum decomposition rate of is shifted to lower values. Moreover, similarly as above, the values of the characteristic temperatures of 5 , 25,50 and $90 \%$ of weight loss were shifted toward lower values.

\section{Conclusions}

The series of PETG-block-PTMO copolymers with different content of flexible PTMO segment were synthesized by means of a two-step melt polycondensation process and characterized by various methods. The rigid segments of PETG as well as the flexible PTMO ones were randomly distributed along the chain. Due to the partial thermodynamic miscibility of the PETG and the PTMO segments, the resulting block copolymers exhibited only one glass transition temperature, which values decrease along with the weight fraction of PTMO segments. On the basis of ${ }^{1} \mathrm{H}$ NMR, SAXS and WAXS, DSC an DMTA results, one can conclude that both phases were thermodynamically miscible and the values of glass transition temperatures decrease along with the content of PTMO flexible segments. The copolymers containing 20 and $30 \mathrm{wt} \%$ of flexible segments exhibit elastomeric property characteristics. Moreover, with respect to the flexible segments content, the temperatures corresponding to $5 \%$ and $90 \%$ weight loss as well as the values of Young's modulus decrease with the increasing flexible PTMO segments content.

\section{Conflicts of interest}

There are no conflicts to declare.

\section{Acknowledgements}

This work is the result of the research project GEKON2/O5/ 266860/24/2016 funded by the National Centre for Research and Development and National Fund for Environmental Protection and Water Management, Poland. Diffraction experiments were performed at BL-11-NCD beamline at ALBA Synchrotron with the collaboration of ALBA staff.

\section{Notes and references}

1 Z. Roslaniec, Polyester thermoplastic elastomers: Synthesis, properties, and some applications, in Handbook of condensation elastomers, ed. S. Fakirov, Wiley-VCH, Weinheim, 2005, ch. 3, p. 77.

2 D. W. Van Krevelen and K. Te Nijenhuis, Properties of Polymers, Elsevier B. V., Amsterdam, 2009.

$3 \mathrm{~J}$. Scheirs and T. Long, Modern polyesters, chemistry and technology of polyesters and copolyesters, Wiley- $\mathrm{VCH}$, Chichester, 2003.

4 R. Saint-Loup, J.-J. Robin and B. Boutevin, Macromol. Chem. Phys., 2003, 204, 970.

5 J. V. Kurian, J. Polym. Environ., 2005, 13, 160.

6 A. Szymczyk, E. Senderek, J. Nastalczyk and Z. Roslaniec, Eur. Polym. J., 2008, 44, 436.

7 G. Li, J. Jiang, J. Jin, S. Yang and C. Wu, J. Macromol. Sci., Part B: Phys., 2006, 45, 639.

8 M. C. E. J. Niesten, J. Feijen and R. J. Gaymans, Polymer, 2000, 41, 8487.

9 X. Guo, J. Xin, X. Lu, B. Ren and S. Zhang, RSC Adv., 2015, 5, 485.

10 D. Hou, J. Xin, X. Lu, X. Guo, H. Dong, B. Ren and S. Zhang, RSC Adv., 2016, 6, 48737.

11 H.-J. Bang, H.-Y. Kim, F.-L. Jin and S.-J. Park, J. Ind. Eng. Chem., 2011, 17, 805.

12 A. Ranade, N. D'Souza, C. Thellen and J. A. Ratto, Polym. Int., 2005, 54, 875.

13 K. Shih and W. Shrinkage, Modeling of Polyester Shrink Film, Polym. Eng. Sci., 1994, 34, 1121.

14 T. Chen and J. Zhang, Appl. Surf. Sci., 2016, 388, 133.

15 C.-L. Liu, W.-T. Sun, W. Liao, W.-X. Lu, Q.-W. Li, Y. Jeong, J. Liu and Z.-H. Zhao, Int. J. Oral Sci., 2016, 8, 246.

16 Y. M. Heo, J. M. Koo, D. K. Hwang, J. G. JaeGal, S. Y. Hwang and S. S. Im, RSC Adv., 2016, 6, 57626.

17 L. Piergiovanni and S. Limbo, Food Packaging Materials, Springer International Publishing, Berlin, 2016, p. 33.

18 S. T. C. Lin, D. S. Musson, S. Amirapu, J. Cornish and D. Bhattacharyya, J. Biomed. Mater. Res., Part A, 2017, 105, 1393.

19 T. Chen, J. Zhang and H. Youa, RSC Adv., 2016, 6, 102778.

20 A. E. Scripa, D. G. Dimitriu and D. O. Dorohoi, J. Mol. Struct., 2017, 1140, 67.

$21 \mathrm{~J}$. Hu, W. Chen, B. Zhao and D. Yang, Constr. Build. Mater., 2017, 131, 411. 
22 A. Kuzminovaa, M. Vandrovcováb, A. Shelemina, O. Kyliána, A. Choukourova, J. Hanuša, L. Bačákováb, D. Slavínskáa and H. Biederman, Appl. Surf. Sci., 2015, 357, 689.

23 R. Schwödiauer, S. Bauer-Gogonea, S. Bauer, J. Heitz, E. Arenholz and D. Bäuerle, Appl. Phys. Lett., 1998, 73, 2941.

24 J. Hu, Y. Li, C. Li, W. Chen and D. Yang, Polym. Test., 2017, 59, 362 .

25 N. Benipal, J. Qi, J. C. Gentile and W. Li, Renewable Energy, 2017, 105, 647.

26 C. Galliot and R. H. Luchsinger, Polym. Test., 2011, 30, 356.

27 L. Charbonneau, M. A. Pola and A. Penlidis, Constr. Build. Mater., 2014, 60, 63.

28 H. Matsukizono and T. Endo, J. Polym. Sci., Part A: Polym. Chem., 2016, 54, 487.

29 A. Kuzminova, A. Shelemin, O. Kylian, A. Choukourov, H. Valentoa, I. Krakovský, J. Nedbal, D. Slavínsk_a and H. Biederman, Polym. Degrad. Stab., 2014, 110, 378.

30 W. Li, Y. Zhai, P. Yi and Y. G. Zhang, Microelectron. Eng., 2016, 164, 100.

31 T. Chen, W. Zhang and J. Zhang, Polym. Degrad. Stab., 2015, 120, 232.

32 K. Lee, J. Lee, B. A. Mazor and S. R. Forrest, Light: Sci. Appl., 2015, 4, 288.

33 B. Li, X. Zhang, Q. Zhang, F. Chen and Q. Fu, J. Appl. Polym. Sci., 2009, 113, 1207.

34 D. Chen, L. Gao, X. Li and Y. Tu, Chin. J. Polym. Sci., 2017, 35, 681.

35 Q. Xu, J. Chen, W. Huang, T. Qu, X. Li, Y. Li, X. Yang and Y. Tu, Macromolecules, 2013, 46, 7274.

36 J. Chen, D. Chen, W. Huang, X. Yang, X. Li, Y. Tu and X. Zhu, Polymer, 2016, 107, 29.
37 K. C. Cole, J. Guèvremont, A. Ajji and M. M. Dumoulin, Appl. Spectrosc., 1994, 48, 1513.

38 R. H. Guo, S. Q. Jiang, C. W. M. Yuen and M. C. F. Ng, J. Mater. Sci.: Mater. Electron., 2009, 20, 735.

39 O. Prasad, L. Sinha, N. Misra, V. Narayan, N. Kumar and J. Pathak, J. Mol. Struct.: THEOCHEM, 2010, 940, 82.

40 T. Chen, G. Jiang, G. Li, Z. Wu and J. Zhang, RSC Adv., 2015, 5, 60570 .

41 A. Szymczyk, Eur. Polym. J., 2009, 45, 2653.

42 S. Paszkiewicz, A. Szymczyk, Z. Spitalsky, J. Mosnacek, E. Janus and Z. Roslaniec, Polimery, 2013, 58, 893.

43 D. Pont, Terathane 1000 polyether glycol, Certificate of analysis, 2000.

44 S. Fakirov, A. A. Apostolov, P. Boeseke and H. G. Zachman, J. Macromol. Sci., Part B: Phys., 1990, 29, 379.

45 H. H. Chuah, Amorphous and Crystalline Polyesters based on 1,4-Cyclohexanedimethanol, in Modern polyester: chemistry and technology of polyesters and copolyesters, ed. J. Scheirs and T. E. Long, Wiley, Chichester, 2004, ch. 7, pp. 267-292.

46 W. Brostow, H. E. Hagg Lobland and M. Narkis, J. Mater. Res., 2006, 21, 2422.

47 W. Brostow, H. E. Hagg Lobland and S. Khoja, Mater. Lett., 2015, 159, 478.

48 A. Dorigato, A. Pegoretti, L. Fambri, C. Lonardi, M. Slouf and J. Kolarik, eXPRESS Polym. Lett., 2011, 5, 23.

49 J. Che, M. Wang, J. Li, S. Guo, S. Xu, Y. Zhang, T. Li and M. Wen, Eur. Polym. J., 2009, 45, 3269.

50 A. Szymczyk and Z. Rosłaniec, Polimery, 2006, 51, 627. 